\title{
EFICACIA DEL CONCRETO Y LA BARITINA COMO ATENUADORES DE RAYOS X. UNA REVISIÓN DE LA LITERATURA
}

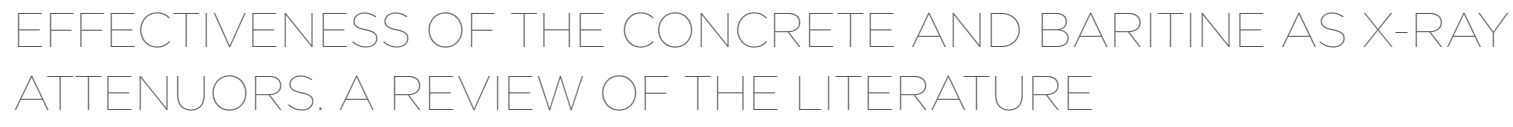

Fiorella Francesca Huañec-Huillca' * (1)

fiorellafrancesca23@hotmail.com

\section{Gustavo Adolfo Fiori-Chíncaro ${ }^{2}$ (i) gfiori@ilaeperu.com}

Artículo recibido: 15/02/2020

Arbitrado por pares

Artículo aceptado: 25/03/2020

\section{* Autor corresponsal:}

Fiorella Francesca Huañec-Huillca fiorellafrancesca23@hotmail.com

Citar como: Huañec-Huillca FF, FioriChíncaro GA. Eficacia del concreto y la baritina como atenuadores de rayos $\mathrm{X}$. Una revisión de la literatura. Rev Cient Odontol 8 (1). (Lima). 2020; 8(1): e010.

DOI: $10.21142 / 2523-2754-0801-2020-010$

\section{RESUMEN}

El presente trabajo busca realizar una revisión de la literatura para dar a conocer al personal ocupacionalmente expuesto a radiaciones ionizantes las ventajas y propiedades del concreto simple y la baritina para justificar su aplicación como materiales de blindaje en el diseño y construcción de muros en áreas donde se utilizan rayos $\mathrm{X}$ de uso odontológico, ya que estos, al no ser utilizados de manera segura, pueden tener efectos perjudiciales en la salud de las personas. En este trabajo también se describen las características generales de los rayos $\mathrm{X}$ que los hacen nocivos para la salud del personal ocupacionalmente expuesto, así como del público en general, por lo que surge en este punto el término de atenuación.

Se encontró poca información sobre el tema, por lo que se desarrolló la relación existente entre ambos materiales y su importancia para dar información que pueda ser útil a todas las personas. Se concluyó que ambos materiales tienen la capacidad de bloquear y reducir la intensidad de la radiación, ya que el concreto actúa como un absorbente para reducir los efectos biológicos de la radiación y la baritina funciona como blindaje basado en el coeficiente de atenuación, por lo que ambos ofrecen una mayor resistencia y, al ser aplicados en conjunto, brindan una mayor protección para aquellas personas que puedan estar expuestas a rayos ionizantes.

Palabras clave: concreto, baritina, atenuación de la radiación, rayos X, blindaje

\begin{abstract}
The safety of the use of radiation in odontology must be guaranteed in order to avoid adverse effects to the health of dental staff and patients. The present study is a review of the literature on exposure to ionizing radiations and the advantages and properties which simple concrete and barite provide as shielding material in wall design and construction in areas of odontology in which $\mathrm{X}$-rays are used.

The general characteristics of X-rays which make them harmful to exposed workers and the general public are described. An attenuation coefficient is the fraction of an $\mathrm{x}$-ray beam that is absorbed or scattered per unit thickness of the absorber.

There is little information on this subject, however, it was found that both concrete and barite are able to block and reduce the intensity of radiation, with concrete acting as an absorbent to reduce the biological effects of radiation and barite acting as a shield. Based on the coefficient of attenuation these two materials provide greater resistance and their combined use allows greater protection against exposure to ionizing rays.
\end{abstract}

Keywords: Concrete, Barite, Radiation attenuation, X-rays, shielding

\footnotetext{
1 Facultad de Estomatología, Universidad Inca Garcilaso de la Vega. Lima, Perú.

2 Instituto Latinoamericano de Altos Estudios en Estomatología (ILAE). Lima, Perú.
} 


\section{INTRODUCCIÓN}

La radiología, a lo largo de su evolución, ha alcanzado beneficios significativos respecto del diagnóstico, sobre todo en el campo de la medicina en general, siempre y cuando se indique y use de manera adecuada; pero la exposición a la radiación ionizante sin hacer uso adecuado de las barreras de atenuación necesarias pone en riesgo la salud tanto del personal ocupacionalmente expuesto como de la población en general $\left(^{1}\right)$.

El blindaje contra la radiación de los rayos $\mathrm{X}$ se fundamenta en el principio de atenuación, que se define como la capacidad de bloquear o reducir la intensidad de radiación por medio de fotoemisión y la dispersión haciendo uso de barreras físicas de protección orientadas a la protección de las personas $\left({ }^{2}\right)$.

Existen diversos organismos internacionales mundialmente reconocidos que se encargan de la elaboración de las normas para el diseño del blindaje. En nuestro país también hay organizaciones que regulan, norman y fiscalizan la edificación adecuada de estas barreras $\left({ }^{3}\right)$.

Con ese fin, se emplean materiales para la construcción de barreras cuyas propiedades y características proporcionan blindaje, como es el caso de la baritina. También llamada barita, es un mineral no metálico, inerte y no contaminante, cuya fórmula química es $\mathrm{BaSO}_{4}$ y que posee un alto peso específico $(4,5 \mathrm{gr} / \mathrm{cc})$, característica a la cual se le atribuye su nombre, que proviene de la palabra griega baros, que significa pesado. La baritina es empleada en la industria de la construcción como blindaje contra rayos $\mathrm{X}$, al ser incorporada en concretos de alta densidad que son colocados alrededor de unidades de rayos $\mathrm{X}$ en hospitales, centrales nucleares y eléctricas $\left({ }^{4}\right)$.

Otro de los materiales utilizados en muchos otros países para el blindaje de rayos $\mathrm{X}$ es el concreto simple, también llamado hormigón, material cuyas principales características son la fortaleza, la durabilidad y el ser relativamente económico de producir y sencillo de formar, en comparación con otros materiales de barrera, por lo que es ampliamente empleado como material básico en obras de construcción, como los hospitales.
El concreto es el resultado de la mezcla de cemento, agregados (que pueden ser grava, gravilla y arena), agua $y$, en algunas ocasiones, aditivos con el fin de modificar o mejorar sus propiedades $\left.{ }^{5,6}\right)$.

Se determinó que, al incorporar minerales pesados como la baritina a un hormigón convencional, se obtiene un concreto de alta densidad, denominación que corresponde a aquellos concretos con densidad superior a los $3000 \mathrm{~kg} / \mathrm{m}^{3}$ y que pueden alcanzar hasta $4800 \mathrm{~kg} / \mathrm{m}^{3}$, por lo que ofrecen una mayor resistencia en comparación con los concretos simples $\left({ }^{7}\right)$.

Por tanto, es importante conocer de manera más profunda las características y propiedades de atenuación de la baritina y del concreto convencional con respecto a la radioprotección, para su uso en el diseño de muros en instalaciones donde se emiten rayos $\mathrm{X}\left({ }^{7}\right)$.

\section{METODOLOGÍA}

Para el desarrollo de esta investigación fue necesaria la búsqueda bibliográfica en Medline, vía PubMed, de diversos artículos y trabajos de investigación entre los años 2004 y 2019 en los que se describen las características de los materiales como el concreto y la baritina como medio de construcción para atenuar rayos $\mathrm{X}$, lo que representa un gran aporte para la bioseguridad. Para ello, se llevó a cabo la búsqueda con las siguientes palabras claves y frases: concreto simple, baritina, atenuación de la radiación, rayos $\mathrm{X}$, radioprotección $\mathrm{y}$ medios de construcción para atenuar rayos $\mathrm{X}$.

\section{RAYOS X Y ATENUACIÓN}

Los rayos $\mathrm{X}$ son un tipo de radiación de ondas electromagnéticas que, a su vez, son ionizantes, debido a que al interactuar con la materia producen la ionización de sus átomos, lo que genera iones $\left({ }^{7,8}\right)$. Los rayos $\mathrm{X}$ se generan mediante el calentamiento de filamentos de tungsteno, lo que origina una nube de electrones que luego es expulsada a gran velocidad para impactar en el punto focal del ánodo, que por lo general está confeccionado con elementos pesados como cobre, 
molibdeno o wolframio. Este proceso da como resultado la generación de rayos $\mathrm{X}$, es decir, radiación característica y radiación por frenado $\left({ }^{8,9}\right)$.

La radiación por frenado es la que se utiliza en el radiodiagnóstico y corresponde del $1 \%$ al $3 \%$ de la radiación generada. Se produce por la descompensación y perdida súbita de la velocidad del electrón con carga negativa, debido a la acción del campo eléctrico del núcleo, el cual tiene carga positiva, con lo cual lo atrae y da como resultado que el electrón varíe su recorrido original y pierda de esta manera su energía cinética. Una fracción de la energía cinética perdida por el electrón en este proceso da lugar a la formación de radiación electromagnética de alta energía $\left({ }^{8,9}\right)$. La radiación característica corresponde al mayor porcentaje de radiación que pasa a convertirse en calor.

Por otra parte, este tipo de radiación es el producto de la colisión de dos electrones, en la cual el primero se desplaza de forma acelerada con dirección al segundo, que pertenece al ánodo localizado próximo al núcleo; esto genera la expulsión de este último que es rápidamente sustituido por el electrón acelerado y genera energía en forma de calor $\left.{ }^{8,9}\right)$.

La interacción de los rayos $\mathrm{X}$ con la materia se puede producir por diferentes fenómenos que sus fotones pueden experimentar con relación a su poder de penetración y la forma en la que interactúan con la materia. Entre estos tenemos los siguientes:

a. Efecto de transferencia, que es la interacción de la materia con el fotón, que la atraviesa en forma de radiación directa.

b. Efecto de absorción, que se refiere a la donación de toda la energía del fotón a la materia para luego desaparecer.

c. Dispersión, en la cual el fotón de rayos $\mathrm{X}$ emerge de la materia en forma de radiación secundaria, como producto de la pérdida de su energía por la desviación en su dirección $\left({ }^{10}\right)$.

La atenuación está definida como la disminución del número de fotones incidentes de los rayos $\mathrm{X}$ cuando penetran en un medio material. La capacidad de atenuación de un material va a depender de la naturaleza de la sustancia del medio, es decir, su número atómico, densidad, espesor y las características de la radiación $\left({ }^{10}\right)$.

Por medio de tres procesos básicos es que se produce la interacción de los fotones con la materia, estos son interrelación fotoeléctrica, el efecto Compton y, finalmente, la creación de pares $\left(^{8}\right)$. La interacción fotoeléctrica es la que se produce entre el átomo de la materia y el fotón, donde este último consume toda su energía con el propósito de remover un electrón de la órbita más cercana al núcleo y dotarla de energía cinética.

Es en ese momento cuando se produce el llamado efecto dominó, como consecuencia de la inestabilidad de la órbita, ya que un electrón será reemplazado por otro de una órbita superior y esto genera la radiación, que es una forma de energía. Las imágenes radiográficas de alta calidad se producen en relación con el efecto fotoeléctrico, ya que este no dará lugar a la formación de radiación dispersa; además, contribuye al mejoramiento del contraste de los diferentes tejidos biológicos.

Por otra parte, los fotones, al incrementar su energía, reducen la probabilidad de ser absorbidos por el efecto fotoeléctrico, lo que da como resultado la disminución del contraste de los tejidos. El efecto fotoeléctrico no es atractivo cuando se hace referencia a la dosis y radioprotección del paciente, ya que todos los fotones que inciden en tejidos liberan energía que es asimilada por el paciente $\left(^{8}\right)$.

Así surge el termino denominado efectos biológicos de la radiación ionizante, que se refiere a las consecuencias no deseadas producto del paso de la radiación a través de un medio biológico, lo que puede producir alteraciones en la estructura o función de las células. Estos efectos biológicos tienen relación con la dosis y el tiempo en que es absorbida $\left.{ }^{(11}\right)$.

Los efectos producidos por la radiación sobre las células pueden ser estocásticos o determinísticos. Los estocásticos son aquellos en los que existe la probabilidad de que se produzca el efecto en función de la dosis, y su severidad es independiente de la dosis, y 
los determinísticos son aquellos en los que la gravedad del efecto y su frecuencia varían en relación con la dosis. La exposición a la radiación puede producir un daño severo en las células que podría alterar su $\mathrm{ADN}$ o incluso conducirlas a la muerte ${ }^{(2)}$.

\section{BARITINA}

La baritina, conocida también como sulfato de bario, espato pesado o barita $\left(\mathrm{BaSO}_{4}\right)$, es un mineral derivado del bario que se extrae mediante un proceso de minería, y tiene como una de sus principales características no ser tóxico. Es muy común y se encuentra, por lo general, junto a la calcita y el cuarzo, y se presenta en la naturaleza como masas cristalinas de color blanco, verdosas, grisáceas o rojizas. Su peso específico varía de 4,3 a 4,6 gramos por cada centímetro cúbico ( $\mathrm{gr} / \mathrm{cc}$ ). En su estado natural, tiene como características ser un material químicamente inerte, de dispersión sencilla y con alta resistencia al calor y a la corrosión. En cuanto a su brillo, puede ser intensificado por medio del blanqueamiento con ácido sulfúrico, y su dureza varía de 2,5 a 3,5 en la escala de Mohs $\left({ }^{13}\right)$.

La mencionada escala fue definida por el geólogo alemán Friedrich Mohs y se basa en la resistencia que ofrecen ciertos minerales al rayado producido por un material más duro sobre un material más blando $\left({ }^{14}\right)$.

El bario, del cual se deriva la baritina, tiene propiedades químicas similares al calcio, solo que posee mayor reactividad. Es uno de los metales alcalinotérreos más estables, su símbolo es $\mathrm{Ba}$, su número atómico es 56 y su peso atómico es 137,3 . No se encuentra en estado puro debido a que reacciona rápidamente con otros elementos del ambiente; por eso, se presenta en forma de compuestos divalentes, como la baritina, el carbonato de bario y el cloruro de bario. Al encontrarse dentro del grupo de los metales es vulnerable a la oxidación, que se produce cuando es expuesto al ambiente y se forman óxido y peróxido de bario. Además, al interactuar con alcohol o agua, puede producir gas hidrógeno $(7,15,16)$.

En cuanto a sus características físicas, se presenta como cristales con forma tubular, aunque también se puede hallar como láminas de conformación poco fina y muy similar al mármol blanco y terroso. Su color es variado: puede ser blanco, blanco amarillento, gris, azul, rojo, café, café oscuro, transparente a translúcido u opaco. Entre sus propiedades físicas, se señala que sus puntos de ebullición y de fusión son de $1140{ }^{\circ} \mathrm{C}$ y $850{ }^{\circ} \mathrm{C}$, respectivamente, con una densidad de $3,5 \mathrm{~g} / \mathrm{ml}\left({ }^{7,17}\right)$.

Las características químicas permiten que los elementos del grupo dos de la tabla periódica, así como el estroncio y el calcio, se relacione de forma muy íntima con los compuestos de bario. A medida que se incrementa el peso atómico de estos elementos alcalinotérreos, como también son conocidos, se produce un aumento en la capacidad de los hidróxidos para disolverse y, al mismo tiempo, se incrementa el peso específico. Por el contrario, la capacidad de solubilidad disminuye en los nitratos, sulfatos y haluros $\left({ }^{7}\right)$. Esta condición favorece su principal característica de atenuación, pues debido a su asociación con otros elementos puede obtener un peso atómico determinado, lo que hace que se le incluya entre los elementos con características de atenuación o barrera para las emisiones de radiación.

La baritina es muy abundante y se encuentra prácticamente en toda la superficie terrestre, por ello ocupa el puesto número 18 respecto de la cantidad con relación a otros minerales. En muchos hacen uso de ella y son aproximadamente 7000 los yacimientos distribuidos alrededor del mundo. En el 2005, China fue el mayor productor mundial de baritina, con un 53\% de la producción, seguida por la India, con el 13\% de la producción, Estados Unidos con el 6\% e Irán con el 4\% $\left({ }^{18-19}\right)$.

En Latinoamérica, uno de los países que registra producción de baritina es el Perú, donde se ha determinado la ubicación de 61 yacimientos, los cuales están distribuidos en las regiones de Junín, Lima, Pasco, La Libertad, Lambayeque, Huánuco y Huancavelica. Estos yacimientos tienen un origen hidrotermal, asociado a vulcanismo de tipo submarino. Las regiones que destacan son Huánuco y Junín, con un 91\% de la producción nacional, la cual está dirigida a los mercados interno y externo. Latinoamérica es el principal destino de esta producción (90\%) y Ecuador es el principal 
comprador. En el Perú, el consumo está cubierto en más del $95 \%$ por la producción interna $\left({ }^{19}\right)$.

Una de las principales causas que genera interés en cuanto a utilidad de la baritina es su peso. Una forma de identificarla es compararla con una piedra que cumpla con las mismas especificaciones y composición parecida, pues el peso de la baritina será 5 veces más que la piedra, lo que la hace de gran utilidad en diversos campos $\left({ }^{18}\right)$. Se estima que, en promedio, el 95\% de la producción de baritina en el mundo se emplea en la perforación de pozos petroleros para formar barros o lodos de perforación; en consecuencia, la producción de este mineral depende de los proyectos de exploración petrolera.E15\% sobrante está destinado a la industria de la pintura para automóviles, como medio de barrera en salas de rayos $\mathrm{X}$ y, como ya se mencionó, para la elaboración de estructuras de concreto de alta densidad $\left({ }^{20}\right)$.

Además, también la baritina, en su estado natural, es empleada en el campo químico para la elaboración de otros derivados que forman parte de cristales y vidrios ópticos, así como para mejorar la blancura en la industria del papel. En el campo de la medicina, es empleada como medio de contraste en la evaluación radiográfica del tracto gastrointestinal, al igual que como relleno para yeso ortopédico y en algunos medicamentos para extender el tiempo límite de caducidad $(7,10,19)$.

La aplicación de la baritina para la formación de concreto de alta densidad en el campo de la construcción es relativamente reciente, como consecuencia del desarrollo de la energía nuclear. Además, este tipo de concreto supone un ahorro económico en comparación con los concretos simples, ya que estos requieren de un mayor espesor para brindar la misma protección. Las construcciones en las que se han hecho uso de este tipo de concreto son muy pocas y específicas, lo que nos muestra el grado de desconocimiento sobre el uso de la baritina para la obtención de los conglomerados necesarios para su producción ${ }^{21}$.

El nivel de protección que ofrece es la misma que la de una lámina de plomo al ser empleada en las proporciones adecuadas. Una lámina de plomo de $1 \mathrm{~mm}$ equivale a $2 \mathrm{~cm}$ de aplanado con baritina y $1,5 \mathrm{~mm}$ a $3 \mathrm{~cm}$, respectivamente, por lo que debe ser aplicada en muros que tengan una superficie firme, capaz de soportar a la baritina por su peso específico, que supera los estándares de materiales de construcción. La altura mínima que se considera es de 2,1 m sobre el nivel del piso; además, es posible colocarla en techos y pisos valorando la ubicación del área como cuando se trata de un primer piso o si colinda con otras áreas inferiores o superiores $\left({ }^{22}\right)$.

\section{CONCRETO SIMPLE}

En muchos casos, los propios muros de las instalaciones donde se hace uso de radiación ionizante pueden proporcionar un blindaje significativo que sustituye la función de otros materiales, como en el caso de los muros de concreto con ladrillo macizo $\left({ }^{23}\right)$.

E1 concreto es una mezcla de cemento, piedra, arena gruesa y agua que experimenta un proceso de endurecimiento conforme se produce la reacción química del agua con el cemento. La resistencia que se indique en los planos de estructuras determinará la cantidad de cada material utilizado en la mezcla. El concreto tiene dos etapas básicas: cuando está fresco y cuando ya se ha endurecido $\left.{ }^{24}\right)$.

El concreto es un material económico y adaptable a cualquier diseño de construcción, que puede actuar como un absorbente para reducir los efectos biológicos de la radiación siempre y cuando presente una fuerza y densidad aceptables $\left({ }^{25-26}\right)$.

Para ello, debe cumplir con un conjunto de requisitos como son la aplicación de las proporciones establecidas para la mezcla, así como debe poder mantener su integridad estructural y efectividad como escudo biológico $\left({ }^{15}\right)$.

La atenuación de los rayos $\mathrm{X}$ depende de la densidad del material de blindaje; se dice que un material de escudo denso con un mayor número atómico es mejor, pues el concreto, al aumentar su densidad mediante la adición de materiales pesados, adquiere un efecto importante en la supresión de estos rayos, lo que contribuye a que los muros de áreas de radiación sean más delgados $\left({ }^{16,23,27}\right)$. 
El cemento es uno de los elementos que forma parte del concreto, el cual es un material fabricado por cocción a elevadas temperaturas a partir de una materia prima que pueda estar compuesta de una mezcla de caliza, arcilla y otros minerales, o simplemente de caliza, al adicionarle un $5 \%$ de yeso que favorece su fraguado. $\mathrm{El}$ proceso de fabricación se inicia con la selección de la materia prima en canteras para proceder a su extracción; esta materia prima es finamente molida y mezclada, para luego proceder a su calentamiento hasta lograr la fusión de los elementos. Este proceso, generalmente, se logra en hornos giratorios de gran tamaño a temperaturas entre $\operatorname{los} 1400^{\circ} \mathrm{C}$ a $1450^{\circ} \mathrm{C}$ para finalmente obtener el clínker, que no es más que la fundición parcial del material. E1 clínker, una vez enfriado, pasa por un proceso de molienda para ser convertido en un fino polvo y es entonces que toma el nombre de cemento. El fraguado del cemento se produce gracias al yeso, que es incorporado en una pequeña proporción durante el proceso de molienda, mientras que los demás agregados pueden ser obtenidos de canteras que, por lo general, son explotaciones a cielo abierto de las que se obtienen rocas industriales, ornamentales o áridos $\left({ }^{28}\right)$.

Para efectos del uso del concreto como barrera de protección para salas de rayos $\mathrm{X}$, la norma técnica del Instituto Peruano de Energía Nuclear (IPEN) menciona que el concreto como estructura de blindaje puede ser utilizado en paredes con un espesor mínimo de $15 \mathrm{~cm}$; sin embargo, no determina las características de ese espacio, por lo que no queda claro si la fabricación debe incluir un tipo determinado de ladrillos (según su uso, de tipo sólido o con huecos) u otro tipo de estructura (piedras, armado de fierros, madera, etc.). Esto sin mencionar el revestimiento que lleva esta pared. Tampoco se mencionan las características de esta capa (grosor, tipo de concreto, tipo de cemento, tipo de mezcla, etc.).

Un trabajo de investigación realizado por la Universidad Nacional de Ingeniería (UNI), titulado "Atenuación de los rayos $\mathrm{X}$ para diagnóstico empleando placas de concreto normal y pesado con baritina”, desarrollado por Gonzales Robles en 2010, concluyó que para el cálculo del espesor de blindaje de concreto normal (densidad 2,3-2,4 $\mathrm{g} / \mathrm{cm}^{3}$ ) para construcciones, se aplica lo establecido en el reporte número 147 del Consejo
Nacional de Protección Radiológica y Medidas (NCRP 147) $\left(^{3}\right)$.

Para ese trabajo se elaboraron dos tipos de placas, una de concreto normal y la otra de mortero con baritina, definiéndose el mortero como una mezcla de arena fina, agua y cemento. Ambas placas tuvieron diferentes espesores y fueron sometidas a disparos de rayos $\mathrm{X}$ con una diferencia de potencial de $100 \mathrm{kV}$ y una cámara de ionización también llamada detector para calcular la cantidad de rayos $\mathrm{X}$ que atraviesan las placas de concreto normal y mortero con baritina.

Con los resultados obtenidos, se elaboró una curva para el cálculo de espesores de mortero con baritina que, al ser comparado con la norma peruana que se basa en el reporte NCRP 147 y las tablas del NIST, dio como resultado que estas cumplen hasta un cierto valor, espesor de $1,8 \mathrm{~cm}$ para la relación concreto/baritina $=$ $1 / 4$ (densidad $3,20 \mathrm{~g} / \mathrm{cm}^{3}$ ) y de $1,9 \mathrm{~cm}$ para la relación $\mathrm{C} / \mathrm{B}=1 / 6$ (densidad $3,30 \mathrm{~g} / \mathrm{cm}^{3}$ ). Por lo tanto, para realizar el cálculo de espesores de mortero con baritina (densidad 3,20-3,30 $\mathrm{g} / \mathrm{cm}^{3}$ ) se debe emplear las curvas obtenidas en esa investigación $\left(^{3}\right)$.

Un ejemplo del uso del concreto de alta densidad fue la construcción de las salas de rayos X en el Hospital Regional de Lambayeque, en Perú, cuyas especificaciones técnicas de arquitectura fueron concluidas por el Gobierno Regional de esa jurisdicción. Se realizó la aplicación en muros y cielo raso de 26 salas de un revestimiento con mortero y barita, el cual fue usado como sustituto del agregado fino.

Las especificaciones de este revestimiento señalaban el uso de 3 capas: la primera fue una capa base, para lo cual se realizó un revestimiento de $1 \mathrm{~cm}$ de espesor cuya proporción de mezcla fue 1:4 correspondiente a cemento y arena; la segunda correspondió a la capa aislante de radiación, para lo cual se empleó la proporción 1:1:4, correspondiente a cemento, arena y baritina, con un espesor no menor de $2 \mathrm{~cm}$; y la capa final se realizó con una proporción 1:5, correspondiente a cemento y arena fina, con $1 \mathrm{~cm}$ de espesor como máximo. Todo esto se elaboró de una forma conveniente para obtener una superficie óptimamente terminada $\left({ }^{29}\right)$. 
El Consejo de Seguridad Nuclear, ubicado en Madrid (España), elaboró una tabla referida a la equivalencia en plomo de materiales como ladrillo de arcilla y concreto más baritina $\left.{ }^{22}\right)$, como se muestra tabla 1 .

\section{CONCLUSIONES}

1. Los efectos nocivos que pueden causar los rayos $\mathrm{X}$ en el organismo humano se previenen mediante el principio de atenuación, el cual depende del material de blindaje y la longitud de onda de los rayos. Para lograr la atenuación, se debe bloquear o limitar la exposición a la radiación a niveles aceptables, mediante el uso de barreras físicas.

2. Entre las características y formas de uso de la baritina como elemento de atenuación contra los rayos $\mathrm{X}$, encontramos que es un material químicamente inerte, de dispersión sencilla, alta resistencia al calor y la corrosión, el cual es vulnerable a la oxidación cuando queda expuesto al ambiente y puede formar óxido y peróxido de bario. Su color es muy variado, sus puntos de ebullición y de fusión son de $1140{ }^{\circ} \mathrm{C}$ y $850{ }^{\circ} \mathrm{C}$, respectivamente, y tiene una densidad de $3,5 \mathrm{~g} / \mathrm{ml}$.

Sus características químicas permiten el aumento de la capacidad de los hidróxidos para disolverse y, al mismo tiempo, que el peso específico del metal se incremente. Por el contrario, la capacidad de solubilidad disminuye en los nitratos y sulfatos. Esta condición favorece sus propiedades de atenuación, pues debido a su asociación con otros elementos puede obtener un peso atómico determinado, lo que hace que se le incluya entre los elementos que se emplean para la atenuación o como barrera para el paso de emisiones de radiación.

3. En cuanto a las características del concreto, se encuentra que es un material económico y adaptable a cualquier diseño de construcción. Así mismo, actúa como un absorbente para reducir los efectos biológicos de la radiación siempre y cuando presente una fuerza y densidad aceptables.

4. El concreto más la baritina puede ser usado como blindaje en muros de áreas donde se emplean rayos $\mathrm{X}$, ya que ambos materiales son empleados como blindaje contra ellos, debido a su coeficiente de atenuación, que puede alcanzar densidades entre 4000 y $4800 \mathrm{~kg} / \mathrm{m} 3$ Por ello, ofrece una mayor resistencia en comparación con los concretos simples, además de que requiere un menor grosor que los muros de concreto simple para cumplir la misma función.

Tabla 1. Equivalencia en plomo de materiales como ladrillo de arcilla y concreto más baritina

\begin{tabular}{|c|c|c|c|c|c|c|}
\hline \multirow{2}{*}{ Material } & \multirow{2}{*}{$\begin{array}{l}\text { Densidad del material } \\
\text { (kg.m-3) }\end{array}$} & \multirow{2}{*}{$\begin{array}{l}\text { Espesor del material } \\
(\mathrm{mm})\end{array}$} & \multicolumn{4}{|c|}{ Equivalente en $\mathrm{mm}$ de plomo según los KV aplicados } \\
\hline & & & 50 & 75 & 100 & 150 \\
\hline \multirow[t]{5}{*}{ Ladrillo de arcilla } & 1600 & 100 & 0,6 & 0,8 & 0,9 & 0,8 \\
\hline & & 200 & 1,4 & 1,7 & 1,9 & 1,7 \\
\hline & & 300 & 2,2 & 2,7 & 3,1 & 2,6 \\
\hline & & 400 & - & 3,8 & 4,5 & 3,7 \\
\hline & & 500 & - & - & - & 4,8 \\
\hline \multirow{7}{*}{ Concreto más baritina } & 3200 & 10 & 0,9 & 1,5 & 1,8 & 0,9 \\
\hline & & 20 & 1,8 & 2,7 & 3,3 & 1,8 \\
\hline & & 25 & 2,3 & 3,3 & 4,0 & 2,2 \\
\hline & & 50 & - & - & - & 4,3 \\
\hline & & 75 & - & - & - & 5,9 \\
\hline & & 100 & - & - & - & - \\
\hline & & 125 & - & - & - & - \\
\hline
\end{tabular}

Fuente: Consejo de Seguridad Nuclear 
Contribución de autoría: Fiorella Francesca HuañecHuillca ha participado en la elaboración del artículo, la recolección de la información, la redacción y aprobación de la versión final. Gustavo Fiori-Chíncaro ha participado como asesor y ha aprobado la versión final.
Fuente de financiamiento: Autofinanciado.

Potenciales conflictos de interés: Los autores declaran no tener ningún conflicto de interés.

\section{REFERENCIAS BIBLIOGRÁFICAS}

1. Ludlow J, Davies Ludlow L, White S. Patient risk related to common dental radiographic examinations. J Am Dent Assoc. 2008;139 (9): 1237-43. doi: 10.14219/jada.archive.2008.0339

2. Akkurt I, Basyigit C, Kilincarslan S, Mavi B. Radiation shielding of concretes containing different aggregates. Cem Concr Compos. 2006; 28 (2): 153-7.

3. Gonzales J.Atenuación de los rayos $\mathrm{X}$ para diagnostico empleando placas de concreto normal y pesado con baritina [Tesis]. Lima: UNI; 2010. Disponible en https://www.researchgate. net/publication/267751536_ATENUACION_DE_LOS_ RAYOS_X_PARA_DIAGNOSTICO_EMPLEANDO_ PLACAS_DE_CONCRETO_NORMAL_Y_PESADO_ CON_BARITINA

4. Romero W. Evaluación del deterioro del revestimiento con baritina para el aislamiento de los cuartos especiales expuestos a rayos $\mathrm{x}$ de la obra "mejoramiento y ampliación de la capacidad resolutiva de los servicios de salud del hospital regional Daniel A. Carrion del distrito de Yanacancha, provincia de Pasco [Tesis]. Pasco: Universidad Nacional Daniel Alcides Carrión; 2018. Disponible en http://repositorio.undac.edu.pe/bitstream/ undac/331/1/T026_48288706_T.pdf

5. Sakr K. Efecto de las altas temperaturas o fuego en las propiedades del hormigón de peso pesado. Effect of high temperature or fire on heavy weight concrete properties. Cem Concr Res. 2005; 35 (3): 590-6.

6. Yura.Manual de construcción. Tomo 1.Lima:2014.Disponible en http://www.yura.com.pe/wp-content/uploads/2018/09/manualde-construccion.pdf

7. Álvarez A. Uso de baritina en hormigones colados para protección radiológica. Guatemala: Universidad de San Carlos; 2013. Disponible en http://biblioteca.usac.edu.gt/ tesis/08/08_3610_C.pdf

8. Alcaraz M. Bases físicas y biológicas del radiodiagnóstico médico. 2.a ed. Murcia: Universidad de Murcia, Servicio de publicaciones; 2003. Disponible en https://books.google.com.pe/ books? id=mtQkRFUmhgs $C \&$ printsec $=$ frontcover\&source $=$ gbs ge_summary_r\&cad $=0 \# v=$ onepage $\& q \& f=$ false
9. Almeida J. Caracterização de argamassas de barita como blindagens contra a radiação $\mathrm{X}$ e determinação experimental dos coeficientes de atenuação desses materiais [Tesis]. Ouro Preto, Brasil: Universidade Federal de Ouro Preto; 2014. Disponible en https://www.repositorio.ufop.br/handle/123456789/5674

10. Miñano UA, Patiño CA. Elaboración de agregados con barita para el diseño de concretos de alta densidad que atenúen los rayos ionizantes [Tesis], Lima: Universidad San Martín de Porres; 2015. Disponible en www.repositorioacademico. usmp.edu.pe/bitstream/handle/usmp/2066/minano_patino. pdf? sequence $=1$ \&isAllowed $=y$

11. Fuentes L, Felipe S, Valencia V. Efectos biológicos de los rayos X en la práctica de estomatología. Rev Haban Cienc Méd. 2015; 14 (3): 337-47.

12. Núñez M. Efectos biológicos de las radiaciones - Dosimetría. Montevideo: Escuela Universitaria de Tecnología Médica, Comité de Tecnólogos de ALASBIMN; 2008. Disponible en https://www.alasbimn.net/comites/tecnologos/material/ Efectos_biologicos_de_las_radiaciones.pdf

13. Samarin A. Use of concrete as a biological shield from ionising radiation. Energy and environmental engineering 2013; 1 (2): 90-7. Disponible en https://pdfs.semanticscholar.org/ de86/8184b4ca4bfc12c865e83b5e0aab639dbce3.pdf.

14. Moreno H, Ibáñez S. La escala de Mohs. Dureza de los minerales. Valencia: Universidad Politécnica de Valencia; 2018. Disponible en https://riunet.upv.es/bitstream/ handle/10251/105177/Moreno\%3BIbañez\%20-\%20La\%20 Escala\%20de\%20Mohs\%3A\%20Dureza\%20de\%201os\%20 Minerales.pdf? sequence $=1$

15. Agencia para Sustancias Tóxicas y el Registro de Enfermedades. Resumen de salud pública. Bario. EE. UU.: US Environmental Protection Agency; 2005. Disponible en https://www.atsdr.cdc. gov/es/phs/es_phs24.pdf

16. Oskarsson A, Nordberg G, Fowler B, Nordberg M. Manual de toxicología de los metales. Vol. II: Metales específicos. Londres: Elsevier; 2015. Disponible en https://www.atsdr.cdc.gov/es/phs/ es_phs24.pdf 
17. Miras A, Galán E. Distribución, características generales y aplicaciones de la barita del suroeste español. Boletin Geológico y Minero 1992; 103 (5): 893-900.

18. Minerales y Rocas. Baritina. Disponible en https://www. mineralesyrocas.com/baritina/

19. Díaz A, Amésquita M, Zedano J, Acosta H. Síntesis geológica, mercado y perspectivas de los minerales de baritina, boratos, sales y azufre en el Perú. Lima: INGEMMET; 2006. Disponible en https://www.ingemmet.gob.pe/documents/73138/170670/S\% C3\%8DNTESIS+GEOL\%C3\%93GICA,+MERCADO+Y+P ERSPECTIVAS+DE+LOS+MINERALES+DE+BARITIN $\mathrm{A},+\mathrm{BORATOS},+\mathrm{SALES}+\mathrm{Y}+\mathrm{AZUFRE}+\mathrm{EN}+\mathrm{EL}+\mathrm{PER} \% \mathrm{C} 3 \%$ 9A/80efb1bb-7237-4b5b-be03-0fa40db16cc7

20. Dirección General de Desarrollo Minero. Perfil de merado de la barita. Ciudad de México; 2014 Disponible en https://www.gob. $\mathrm{mx} / \mathrm{cms} /$ uploads/attachment/file/5568/pm_barita_2014.pdf.

21. Martínez L. Hormigón pesado. Una mirada actual de su empleo como blindaje. 2019 Disponible en https://www.monografias. com/docs115/hormigon-pesado-blindaje/hormigon-pesadoblindaje.shtml.

22. León barita. Barita para mi sala de rayos X. 2019. Disponible en https://www.leonbarita.com/barita/

23. Consejo de Seguridad Nuclear. Aspectos técnicos de seguridad y protección radiológica de instalaciones médicas de rayos X para diagnóstico. Madrid; 1990. Disponible en http://piramidenormativa.sne.es/Repositorio/CSN/GSG05.11\%20Aspectos $\% 20$ tecnicos $\% 20 \mathrm{de} \% 20$ seguridad $\% 20$ $\mathrm{y} \% 20$ proteccion $\% 20$ radiologica $\% 20 \mathrm{de} \% 20$ instalaciones $\% 20$ medicas\%20de\%20rayos\%20X\%20para\%20diagnostico\%20 OCR.pdf
24. Aceros Arequipa. Manual del maestro constructor. 2019. Disponible en http://www.acerosarequipa.com/manual-delmaestro-constructor/materiales-de-construccion/concreto.html

25. Mahmound A. Improving X-Ray radiation resistance of concrete used in radio-diagnostic centers [Tesis]. Islamabad, Pakistán: The Islamic University-Gaza Faculty of Engineering; 2015. Disponible en https://library.iugaza.edu.ps/thesis/117006.pdf

26. Rezaei Ochbelagh AS. Investigation of gamma-ray shielding properties of concrete containing different percentages of lead. Applied Radiation and Isotopes 2012; 70 (10): 2282-6. Disponible en https://www.sciencedirect.com/science/article/ abs/pii/S0969804312003855?via\%3Dihub.

27. Abdelnabi A, Odi H, Mohammad H, Mobark. The effect of replacements $30 \%$ for each concrete component by iron filling in concrete on attenuation properties. American Research Thoughts 2015; 2 (1): 3034-45. Disponible en https://www. researchgate.net/publication/308963387_THE_EFFECT_ OF_REPLACEMENTS_30_FOR_EACH_CONCRETE_ COMPONENT_BY_IRON_FILLING_IN_CONCRETE_ ON_ATTENUATION_PROPERTIES

28. Napa L. Estudio de las propiedades físicas del concreto pesado con agregado grueso de baritina y cemento Portland Tipo I [Tesis]. Lima: Universidad Nacional de Ingeniería; 2002.

29. Mondragón K. Comparación entre el concreto convencional y el concreto con barita en polvo como sustituyente del agregado fino. [Tesis]. Pimentel, Perú: Universidad Señor de Sipan; 2016. 